\title{
Sobre la naturaleza de las cualidades expresadas por el Predicativo con verbos estativos en latín
}

\author{
Concepción Cabrillana
}

(University of Santiago de Compostela)

\section{On the Nature of Qualities Expressed by Praedicativa with Stative Verbs in Latin}

\begin{abstract}
This article examines the type of qualities that can be expressed by the Praedicativum in cases in which this secondary predicate is subject-oriented. More specifically, I will discuss the apparently contradictory situation that occurs in contexts with stative verbs and verbs with a semantic notion of permanence and the non-permanent quality attributed to the Praedicativa in a general way. Through a corpus study, I show (i) how the position held so far by researchers should be clarified, and (ii) the explanations that can be made for the results that deviate from the usual trend. Among other factors, the particular nature of the semantics of the predicates under study plays a decisive role in the analysed question.
\end{abstract}

\section{Keywords}

Latin language, Praedicativum, stative verbs, (non)permanent quality

Este trabajo se realiza en el marco del Proyecto de Investigación FFI2017-83310-C3-2-P, denominado "Interacción del léxico y la sintaxis en griego y en latín: el predicativo y los procesos de copularización", financiado por el Ministerio de Economía y Competitividad del Gobierno español y por Fondos FEDER. Quiero dejar constancia de mi agradecimiento a los dos revisores anónimos de este trabajo por las sugerencias que han aportado. 


\section{Introducción: objeto de estudio, estructura y corpus del trabajo}

En torno a la problemática función del Predicativo ${ }^{1}(P)$ existe una cuestión apenas puesta en duda, sobre la que se han expresado algunas afirmaciones: se trata del tipo de cualidad que puede ser expresada por el término que funciona como P. Así, se constata un acuerdo bastante generalizado a la hora de entender que el $\mathrm{P}$ ha de expresar cualidades transitorias o 'de estadio', ancladas temporalmente en el proceso verbal que se desarrolla. ${ }^{2}$ Esta característica ha llevado a ciertos investigadores ${ }^{3}$ a entender que los $\mathrm{P}$ serían incompatibles con verbos de naturaleza estativa; apoyan esta postura, entre otras razones, en el hecho de que el predicado mismo expresa ya que el Sujeto $(S)$ se encuentra en un estado, de forma que los predicados estativos indicarían un proceso no realizado ocasionalmente y se configuran lingüísticamente como estables, concebidos al margen de realizaciones temporales o espaciales. En un trabajo anterior ${ }^{4}$ se mostraban algunos contraejemplos de esta afirmación y se exponía la necesidad de realizar un estudio más detenido sobre la cualidad no permanente del $\mathrm{P}$ que se suele aducir. Aquí se enmarca el propósito de este trabajo, en el que se efectuará además un estudio de corpus: la cuestión fue solo aludida tangencialmente en el estudio al que se ha hecho referencia y allí se proponía la realización de investigaciones sobre conjuntos de textos para examinar con mayor detalle varias de las cuestiones oscuras o problemáticas que afectan a la función de $\mathrm{P}^{5}$

Más concretamente, se estudiará la cuestión aludida en cuatro predicados de naturaleza estativa (maneo, permaneo, remaneo y sto); algunos de ellos albergan intrínsecamente una noción de permanencia, si bien dicha permanencia puede ser temporal, como muestran los siguientes ejemplos:

(1a) postero die patenti itinere Priaticus campus eos excepit, triduumque ibi frumentum accipientes manserunt ${ }^{6}$ ("al día siguiente, con el camino despejado, los acogió la llanura Priática, donde permanecieron tres días haciendo acopio de trigo", ${ }^{7}$ Liv. 38,41,8)

1 De manera general, se entiende aquí por Predicativo el constituyente en principio no argumental que denota la manera en la que la entidad involucrada en el proceso verbal desarrolla dicho proceso mientras este tiene lugar: cf. Cabrillana (2019a: § 2).

2 Sobre este último punto, cf. e.g., Halliday (1967) y Rothstein (1983) apud Gumiel (2008: p. 171), Demonte \& Masullo (1999: p. 2474), Schultze-Berndt \& Himmelmann (2002), Gumiel (2005: pp. 21, 31, 35; 2008 : pp. 173, 177), Tarriño (2009: p. 258), Pinkster (1995: p. 196; en prep.: p. 25); agradezco a H. Pinkster el haberme proporcionado este material en preparación en su versión pre-final, inédito aún a la hora de confeccionar este trabajo.

3 Cf., entre otros, Demonte \& Masullo (1999: p. 2475), De Miguel \& Fernández Lagunilla (2002: p. 222), di Tullio (2002: p. 14), Gumiel (2005: p. 36). Cf. al respecto Silvagni (2018).

4 Cabrillana (2019a: $§ 3.2)$.

5 Cf. Cabrillana (2019a: $\$ \$ 3.2$ y 5).

6 Los textos latinos corresponden a los de la Base de Datos LASLA o, cuando esta base no incluye los textos analizados, a los que utiliza el PHI: se trata de las dos herramientas digitales utilizadas en la labor de localización de los ejemplos que constituyen el corpus de este análisis.

7 Si no se indica lo contrario, las traducciones están tomadas de las correspondientes a la Biblioteca Clásica Gredos. 
(1b) cum aedificandum fuerit, ante biennium ea saxa non hieme sed aestate eximantur et iacentia permaneant in locis patentibus ("cuando haya que acometer una obra, dos años antes se extraerán las rocas -no en invierno sino en verano- y se dejarán expuestas a cielo abierto", Vitr. 2,7,5)

(1c) cessitque res prospere, quando captis castris lectica eius, quasi ibi cubans remansisset (sc. Augustus), concursu hostium confossa atque lacerata est ("y más le valió, pues, cuando tomaron su campamento, su litera fue acribillada y despedazada por los enemigos, que se lanzaron sobre ella pensando que se había acostado allí (sc. Augusto)”, Suet. Aug. 91,1,5)

(1d) igitur accita est (sc. Servilia) in senatum, steteruntque diuersi ante tribunal consulum ("el caso fue que se la hizo comparecer (sc. Servilia) ante el senado, y quedaron en pie el uno frente al otro ante el tribunal de los cónsules”, Tac. ann. 16,30)

En todos los casos anteriores, el propio contexto da a entender que la permanencia en un estado concreto no se da durante toda la existencia de la entidad de la que tal estado se predica; esta circunstancia puede estar explicitada en algunos casos, además, por determinados elementos léxicos (triduum [1a], ante biennium [1b]). Se trata por tanto, de $\mathrm{P}$-codificados por participios o adjetivos- que expresan, en efecto, modos de estar ${ }^{8}$ o procesos verbales ${ }^{9}$ transitorios.

Sin embargo, cabe preguntarse cuál será la situación específica y completa en predicados que, per se, tienden a expresar una noción semántica de permanencia que en ocasiones será atélica. ${ }^{10}$ Aunque ello no es enteramente así en el caso de sto, es este un predicado que se encuentra entre los que Pinkster (en prep.: 3) considera como verbos con los que es frecuente que aparezcan P. ${ }^{11}$ En todo caso, pueden tener lugar situaciones que planteen problemas, precisamente por la posibilidad de que se produzca una suerte de "choque de contrarios": la confluencia de procesos verbales permanentes y $\mathrm{P}$ no permanentes. Así, el presente trabajo intentará responder a las cuestiones que surjan de esta situación a primera vista contradictoria. Al hilo de esta situación, conviene hacer un breve apunte sobre la situación pragmática del $\mathrm{P}$, que con no poca frecuencia, y de modo más específico cuando es delimitado por un complemento de Tiempo en los contextos que se estudian aquí, aporta información focal. ${ }^{12}$

Por otro lado, la elección de este tipo de predicados responde, además, al marco de investigación más amplio en el que se inserta este trabajo, como es el del estudio de los

8 En (1b)-(1d).

9 En (1a), accipientes denota acción.

10 Se entiende por permanencia "atélica" la que se da cuando no parece existir o conocerse un límite inicial o final del proceso verbal.

11 Aunque el lingüista holandés señala que la construcción de P no está semánticamente restringida, observa que esta es frecuente con verbos "of standing, moving, finding and perceiving" (Pinkster, en prep.: p. 3).

12 Resulta lógico que cuando un verbo estático se combina con un $\mathrm{P}$ que expresa una situación permanente, el P aporte información relevante. Cf. a este respecto, p.e., (10a) y su nota correspondiente, o también (11), donde el autor realiza un juego de palabras que subraya dicha focalidad del P (inuita / in uita). Esta cuestión de la relevancia pragmática del P fue apuntada ya en Cabrillana (2010: esp. § 3) y es objeto de estudio específico en Cabrillana (2019b: § 3.3). 
procesos de copularización de distintos predicados. ${ }^{13}$ Esto justifica, asimismo, que solo se investiguen aquí los $\mathrm{P}$ que se encuentren orientados al $\mathrm{S}$, tanto si se construyen en concordancia como si esta está ausente. ${ }^{14}$

Así pues, se tratará de examinar si la expresión de cualidades no permanentes, en principio habitual en el $\mathrm{P}$, opera del mismo modo en verbos de estado y, entre ellos, en algunos que pueden poseer la noción de permanencia - atélica o no- como contenido semántico principal.

La estructura del trabajo se organiza como sigue: se partirá de una breve exposición de lo que recoge la investigación sobre el punto a tratar (\$2); a continuación, se presentarán las diversas situaciones que un estudio de corpus prosístico manifiesta: casos que siguen la línea característica de las situaciones excepcionales distinguidas en la investigación anterior (§ 3.1), casos que se apartan de esa línea (§ 3.2) y contextos especialmente particulares (§ 3.3). El trabajo se cerrará con una recopilación de las conclusiones más significativas.

Como corpus objeto de estudio se han recogido los casos de Predicativos con los verbos maneo, permaneo, remaneo y sto en los siguientes autores y obras: Cato (agr.), Varro, Cic. (Arch., Att., Balb., Caecin., Cael., Catil., Cato, Cluent., div. in Caec., dom., Flac., Font., har., resp., Lael., leg. agr., Lig., Manil., Mil., Mur., nat. deor., off., Phil., Pis., Planc., prov., Quinct., Rab. perd., Rab. post., p. red. ad Quir., p. red. in sen., rep., S. Rosc., Sest., Sull., Tusc., Vatin., Verr.), Caes. (Gall., civ.), Sall. (Cat., Iug.), Vitr., Liv. 1-45, Vell., Sen. (contr., suas.), Sen. (dial., epist., nat.), Curt., Plin. (nat.), Colum., Quint. (inst.), Tac. (ann., hist.), Petron., Suet., Plin. (paneg., epist.), Apul. (met.). Pese a esta amplitud, el número total de ejemplos sobre los que realizar el estudio concreto no es especialmente alto (178x); sin embargo, y precisamente por lo dilatado del campo de análisis, las cantidades resultantes pueden considerarse suficientemente representativas. La búsqueda de casos analizables y su examen se han realizado caso a caso.

\section{Aportaciones precedentes y situación en el corpus}

Como se ha dicho, las posturas generalizadas en la investigación precedente se inclinan por defender que la naturaleza de la cualidad expresada por el $\mathrm{P}$ ha de ser no permanente, lo cual impediría que apareciesen $\mathrm{P}$ con Verbos $(V)$ que implican estado \pm permanente; cuando no es así, se ha indicado que no se tiene explicación para tales ejemplos aunque se reconoce que se trata de casos diferentes de los habituales; ${ }^{15}$ también se ha propugnado que se trata de casos en los que se produce una desemantización por parte del V que pasaría así a comportarse de modo copulativo. Estas son, en esencia, las posturas que defiende, entre otros, Gumiel (2005: p. 34; 2008: p. 182, n. 9).

13 Cf. nota al título del artículo y Cabrillana (2015; 2018).

14 Cf., p.e., (7), donde aparecen dos $\mathrm{P}$ en concordancia con el S en nominativo (admirabilis, celsus) y otro P en genitivo (magnitudinis uerae); asimismo, en (10a) puede verse un $\mathrm{P}$ codificado por un sintagma preposicional en ablativo (sine uitiis).

15 Situaciones que se darían en ejemplos como "el niño nació sordo" o "ella murió joven"; cf. Halliday (1967: p. 78) o Martínez Linares (2003: p. 435). 
Pinkster es algo más explícito en el caso de la lengua latina para la que, aunque se decanta asimismo por la preferencia de las cualidades no permanentes expresadas por los $\mathrm{P}$, reconoce una mayor libertad en los poetas; estos muestran construcciones en las que el $\mathrm{P}$ puede expresar tamaño (ingens) o sustancia (aeneus). ${ }^{16} \mathrm{El}$ autor (en prep.: p. 5) señala también que pueden aparecer $\mathrm{P}$ que denoten propiedades permanentes, sobre todo si indican una condición física estativa, edad o posición socio-económica, ${ }^{17}$ y ello no restringido exclusivamente al género poético, si bien no se da con demasiada frecuencia.

De acuerdo con ello, en un corpus enteramente prosístico como el elegido para esta investigación, los ejemplos que rompen la tendencia de expresión de cualidades no permanentes por parte de los $\mathrm{P}$ deberían ser inexistentes o escasos. Pues bien, de los 178 casos del total del corpus, en 31 ocasiones $(17,41 \%)$ los $\mathrm{P}$ denotan cualidades que pueden considerarse permanentes; se trata de una cantidad nada desdeñable - cercana al $20 \%$ - y tanto más significativa cuanto que, como se acaba de recordar, ninguno de los casos responde a un uso poético.

Procede ahora estudiar las características de cada uno de estos casos y el contexto en el que se presentan.

\section{Características y contextos de la expresión de cualidades no permanentes}

La organización de los contenidos de este apartado se propone avanzar de menor a mayor singularidad en la presentación y explicación de los distintos contextos. Así, se verán primero los casos que se encuadran en las excepciones que recogía Pinkster para aquellos casos de género mayoritaria pero no exclusivamente poético (P que denoten condición física estativa, edad o posición socio-económica: § 3.1); a continuación se tratarán los casos que quedan fuera de estas coordenadas semánticas $(\$ 3.2)$ y, por último, los textos en los que los diversos $\mathrm{P}$ participan de las dos características anteriores o bien resultan menos claros (§3.3).

\subsection{Casos excepcionales distinguidos en la investigación anterior}

Quizá la nota más llamativa de estos casos - en los que el P denotaría condición física estativa, edad o posición socio-económica- es precisamente su escasa aparición, cuando muy posiblemente se habría esperado lo contrario: sólo 5 casos del total de 31 . Por otro lado, en todos los ejemplos el tipo de P elegido expresa una misma circunstancia -la condición física estativa-, como se ejemplifica en (2):

16 Cf. Pinkster (en prep.: p. 9).

17 Cf. Cabrillana (2019a: § 3.2). 
(2) inde omne corpus, ubi nimius ardor aut humor, aut interit aut, si manet, sterile ("from this fact, every body, when there is excessive heat or excessive moisture, perishes, or if it survives, is barren", ${ }^{18}$ Varro rust. 5,61)

Esta circunstancia -la expresión de una cualidad física estativa por parte del $\mathrm{P}$ - puede verse también justificada por la naturaleza del S y sus condiciones, en principio inmutables de por sí; los ejemplos se producen no solo con los verbos que tienen un contenido semántico de permanencia de modo más inherente (3a) sino también con sto (3b), si bien el contexto en el que aparece hace entender una acepción 'existencial' de ese verbo: ${ }^{19}$

(3a) contra Messenii ueterem inter Herculis posteros diuisionem Peloponnesi protulere, suoque regi Denthaliatem agrum in quo id delubrum cessisse; monimentaque eius rei sculpta saxis et aere prisco manere ("en contraposición los mesenios alegaron la antigua división del Peloponeso entre los descendientes de Hércules y que a su rey le había correspondido el campo Dentaliate, lugar donde dicho santuario se encontraba. Que quedaban recuerdos de este suceso, esculpidos en piedras y en bronces antiguos", Tac. ann. 4,43,2)

(3b) stat moles abscisa in profundum, frequentibus exasperata saxis quae aut elidant corpus aut de integro grauius impellant ("allí se alza una mole de piedra con una hendidura profunda y muy escabrosa, llena de incontables rocas contra las cuales los cuerpos tanto quedan destrozados como salen rebotados con gran fuerza”, Sen. contr. 1,3,3)

\subsection{Casos excepcionales no contemplados en la investigación anterior}

Si en el apartado previo se destacaba la sorprendente escasez de ese tipo de ejemplos, en este hay que llamar la atención sobre la circunstancia contraria: los P que aun expresando una cualidad permanente no o no solo denotan condición física estativa, edad o posición socio-económica son clara mayoría (casi un 81\% [= 25x]). Así sucede, por

18 Cuando no se cuenta con una traducción castellana de la Biblioteca Clásica Gredos o suficientemente actualizada y satisfactoria, se ha preferido acudir a las que ofrece la Loeb Classical Library. Se prefiere no proponer traducciones personales para no condicionar la interpretación de los textos, y ello, aun a riesgo de que no siempre las traducciones reflejen de manera fiel el sentido del original latino y los matices de su construcción sintáctica. Es, precisamente, lo que ocurre en la interpretación de este ejemplo, que podría entender que el verbo de sterile fuera una cópula elidida; sin embargo, creo que el proceso verbal que afecta a sterile es, en efecto, el que denota manet; de este modo, una traducción viable sería "si sigue existiendo, lo hace en condición de esterilidad". Creo pues, que la traducción "is barren” es equívoca.

19 Cf. Cabrillana (2018: pp. 617-618). Podría quizá pensarse que elementos como sculpta y exasperata en (3a) y (3b) respectivamente no fueran tanto $\mathrm{P}$ como Atributos del S, como de hecho lo es abscisa; en efecto, existe una frontera difícil de trazar en determinados casos entre estas dos funciones. Con todo, la utilización combinada de diversos tests como los de la pronominalización, la pregunta parcial, la conmutación por una oración adverbial o el test de facio pueden ayudar en cierta medida en la resolución de ejemplos ambiguos. La cuestión es objeto de un estudio independiente que se encuentra actualmente en su fase final de realización: "Predicativo y Atributo en latín: algunos criterios de diferenciación"; cf. especialmente, §§ 3.1-3.2. La aplicación de esos criterios a estos ejemplos proporcionaría más evidencias de que sculpta y exasperata son, en efecto $\mathrm{P}$, y no Atributos. Con todo, en algunas ocasiones faltan pruebas formales absolutamente definitivas y solo puede llegarse a aducir más razones en favor de una interpretación que de otra. 
ejemplo, en (4), donde sollemne $e^{20}$ se refiere a una práctica habitual con un principio pero sin final conocido, esto es, perpetua:

(4) mansit certe sollemne ut quandoque idem prodigium nuntiaretur feriae per nouem dies agerentur ("lo cierto es que quedó como una práctica consagrada el que, cada vez que se anunciaba un prodigio similar, se celebrase una fiesta de nueve días”, Liv. 1,31,4)

En el siguiente ejemplo es posible entender que los elementos con función de P expresan Manera, ${ }^{21}$ algo muy propio de la función del P; la cualidad permanente expresada por tales elementos provendría más bien de la semántica verbal durativa: ${ }^{22}$ se entiende de nuevo que la condición expresada por el $\mathrm{P}$ tiene un principio pero la intención es que no tenga final:

(5) itaque claues portarum reddi sibi aequum censent: bonis sociis fidem suam maximum uinculum esse et ita sibi populum Romanum senatumque gratias habiturum, si uolentes ac non coacti mansissent in amicitia ("que consideran justo, por consiguiente, que se les devuelvan las llaves de las puertas de la ciudad: el vínculo más fuerte entre unos buenos aliados es la lealtad, y ellos tendrán el reconocimiento del senado y el pueblo romano si se mantienen en su amistad voluntariamente y no por la fuerza", Liv. 24,37,7)

Además de la posibilidad de expresar manera, los $\mathrm{P}$ con los verbos estudiados pueden expresar también cantidad. El siguiente ejemplo ilustra esta circunstancia; aunque el $\mathrm{P}$ aludido -solum - pudiera aisladamente tomarse también como adverbio, ${ }^{23}$ creo que tanto el contexto como su posición en la predicación abogan por entenderlo como adjetivo: ${ }^{24}$

(6) per quadriduum Cremona suffecit. Cum omnia sacra profanaque in igne considerent, solum Mefitis templum stetit ante moenia, loco seu numine defensum ("durante cuatro días fue suficiente Cremona. Cuando todo lo sagrado y lo profano quedó sumido en el fuego, solo

20 Puede considerarse que esta palabra se encuentra aquí sustantivada; en esos casos, como afirma Givón (1990: I, p. 51), los conceptos expresados por los sustantivos suelen ser más estables en el tiempo que las cualidades que acostumbran a denotar los adjetivos.

21 El hecho de que habitualmente se pueda conmutar el P por un adverbio que claramente expresa esta Función Semántica -ita-, daría cuenta de la cualidad del P desde el punto de vista semántico.

22 Algo en efecto determinante en el caso de los verbos estudiados. Con todo, la información que aporta el contexto es también decisiva en muchos casos para dotar de una naturaleza permanente a la cualidad expresada por el $\mathrm{P}$.

23 Sobre las posibilidades de que esta clase de palabra funcione como P, cf., entre otros, Kühner \& Stegmann (1912: I, p. 234), Hofmann \& Szantyr (1965: II, 2, p. 425), Fugier (1983), Vester (1983), Gumiel (2005: pp. 38, 65-66, 76), Tarriño (2009: p. 259), Pinkster (1983; 1995: pp. 202, 205; 2015: pp. 859, 861; en prep.: pp. 28-31), Himmelmann \& Schultze-Berndt (2005: pp. 2-15). Un resumen de las posturas al respecto puede verse en Cabrillana (2019a: $\$$ 4.1-4.2).

24 Así lo entiende también la interpretación inglesa: "or four days did Cremona supply food for destruction. When everything sacred and profane sank into the flames, there stood solitary outside the walls the temple of Mefitis, protected by either its position or its deity"; "solitary" solo puede ser sustantivo o adjetivo, que es el caso ante el que nos encontraríamos. Por otro lado, omito aquí la discusión sobre la posibilidad de tener un segundo $\mathrm{P}$ en defensum, dado que podría interpretarse también como Aposición, una de las construcciones relacionadas con el $\mathrm{P}$ con las que en ocasiones resulta difícil trazar una frontera distintiva nítida (cf. Cabrillana 2019a: § 4.3). 
permaneció en pie el templo de Mefitis, delante de las murallas, protegido por el lugar o por la divinidad", Tac. hist. 3,33)

Por último, existen también casos que constatan que es posible que los $\mathrm{P}$ denoten cualidad permanente y que expresen contenido evaluativo, algo, en principio, más frecuente en poesía. ${ }^{25}$ La cualidad atélica de los $\mathrm{P}$ parece clara en ejemplos como el siguiente:

(7) est, mi Lucili, uerus et rebus, non artificiis philosophus. In edito stat admirabilis, celsus, magnitudinis uerae ("tal es, querido Lucilio, el filósofo verdadero, por su comportamiento, no por sus argucias. Está situado en la altura, admirable, sublime, con auténtica grandeza", Sen. epist. 111,3)

\subsection{Casos particulares}

Este apartado aborda casos en los que se dan ciertas situaciones particulares, bien por la excepcionalidad de las mismas, bien por su condición algo más problemática o ambigua.

Por un lado, se encuentran contextos en los que se suceden varios $\mathrm{P}$, algunos de los cuales pueden denotar una cualidad permanente y otros no lo hacen de manera tan clara; es lo que ocurre en (8):

(8) itaque tum, cum uigilando necabatur, erat in meliore causa, quam si domi senex captiuus, periurus consularis (sc. Atilius Regulus) remansisset ("and so even then, when he was being slowly put to death by enforced wakefulness, he enjoyed a happier lot than if he had remained at home an aged prisoner of war, a man of consular rank forsworn”, Cic. off. 3,100)

El contexto de este ejemplo deja ver que se trata de una situación hipotética, pero al mismo tiempo, el Sujeto -Marco Atilio Régulo- era ya en ese momento senex y cónsul por segunda vez; ciertamente la condición de senex tiene inicio pero no final puesto que a partir de un momento se mantiene hasta el término de la vida del hombre y de la misma forma se comportaría la condición hipotética de captiuus, dado el contexto en que aparece, ${ }^{26}$ de otra parte, el estatuto de consularis ciertamente era habitualmente temporal, pero no en la misma medida que el de periurus: una vez cometida la falta que hace a la persona acreedora de tal condición, dicha condición permanece unida de alguna manera a la misma persona: nadie le puede desprender de haber quebrantado maliciosamente un juramento.

En el siguiente ejemplo, el $\mathrm{P}$ es todo un sintagma nominal, pero es el adjetivo correspondiente -hereditarium - el que le otorga una connotación de permanencia atélica al proceso verbal denotado por remansisse:

(9) Harpasten, uxoris meae fatuam, scis hereditarium onus in domo mea remansisse ("sabes que Harpaste, la sirvienta boba que tenía mi mujer, ha permanecido en mi casa como una carga hereditaria”, Sen. epist. 50,2)

25 Cf. Pinkster (en prep.: p. 6).

26 I.e., dando a entender la condición de prisionero de por vida. 
Otra forma mediante la que la lengua provee de la cualidad permanente a un P que expresa manera es mediante la adición de expresiones que poseen esa atelicidad, como pueden ser adverbios durativos - (10a) - o sintagmas preposicionales sin término temporal, o al menos sin que posean alguno de sus límites: en (10b) se desprende que la cualidad de inuictum puede tener cierto término final, pero no inicial:

(10a) namque ei materiae nec caries nec tempestates nec uetustas potest nocere, sed ea et in terra obruta et in aqua conlocata permanet ${ }^{27}$ sine uitiis utilis sempiterno ("y esa madera, ciertamente, no la pueden perjudicar ni la pudrición ni las inclemencias ni el envejecimiento, sino que, lo mismo enterrada que sumergida en agua, resiste eficazmente sin deteriorarse nunca", Vitr. 1,5,3)

(10b) at hercule aliquot annos continuos ante legem Gabiniam ille populus Romanus, cuius usque ad nostram memoriam nomen inuictum in naualibus pugnis permanserit, magna ac multo maxima parte non modo utilitatis sed etiam dignitatis atque imperi caruit ("al contrario -ipor Hércules!- durante algunos años seguidos, antes de la promulgación de la ley Gabinia, ese mismo pueblo romano, que hasta nuestros tiempos había conservado fama de invicto en las luchas por mar, se vio privado de una gran parte -mejor dicho, de la mayor- no sólo de sus ingresos sino aun de su dignidad y de su imperio", Cic. Manil. 54)

Un último caso particular en la línea del ejemplo anterior es (11), donde se produce un singular juego de palabras y sonido. El contexto aclara que se trata de la situación en la que acaba la esposa de Tlepólemo después de la muerte de su marido:

(11) sed aegre manibus erepta suorum inuita remansit (sc. nupta) in uita ("retirada de allí (sc. la esposa) a duras penas por intervención de los suyos, permaneció en el mundo de los vivos sin ganas de vivir", Apul. met. 8,6,21)

\section{Conclusiones}

A lo largo de este trabajo se han mostrado diversas realidades que contravienen o matizan posturas asumidas por distintos investigadores en la cuestión objeto de estudio. Por un lado, y frente a lo que se venía sosteniendo hasta el momento, la necesidad de que el $\mathrm{P}$ exprese cualidades no permanentes no es universal, sino que posee algunas restricciones. Así, y concretamente en predicados de estado $(s t o)$ y en aquellos que poseen además una noción semántica de permanencia (maneo, permaneo, remaneo), aparecen construcciones con $\mathrm{P}$ en las que este designa una cualidad permanente en una proporción relativamente significativa; el dato es tanto más revelador puesto que las excepciones se han reconocido básicamente en contextos poéticos, y en esta investigación se ha trabajado siempre sobre textos de tipo prosístico.

27 Otros manuscritos ofrecen la forma en plural: permanent. Independientemente de ello, el ejemplo resulta particularmente interesante: de un lado, obruta y conlocata son P de tipo más bien concesivo (cf. Martínez Linares 2003: p. 436) y, debido a ello, se establece un contraste entre las condiciones adversas para la conservación de la madera, pese a las cuales, esta permanece inalterable; esto lleva a entender que la relevancia informativa recaería en este caso precisamente en el P y sus adjuntos. 
Por otro lado, el estudio de corpus ha permitido demostrar que las situaciones que se aducían como excepcionales a la hora de que el $\mathrm{P}$ denotara cualidades permanentes - esto es, aquellas en las que el $\mathrm{P}$ expresaría con frecuencia condición física estativa, edad o posición socio-económica- son, con mucho, las menos frecuentes; solo aparecen ejemplos en los que el P manifiesta condición física estativa, y ello debido, en la mayoría de los casos, a que la entidad de la que se predica la cualidad posee una naturaleza frecuentemente inanimada y no sujeta a cambio controlado (p.e., rocas, estatuas, plantas).

Los casos más numerosos son, curiosamente, aquellos que se apartan de las excepciones señaladas por la investigación precedente, de forma que los $\mathrm{P}$ que acompañan a los predicados estudiados aquí expresan más habitualmente manera, cantidad o contenido evaluativo. A su vez, el hecho de que dichos predicados se comporten de este modo, por así decir, atípico, se encuentra en buena medida justificado precisamente por la especificidad del contenido semántico de estos: sobre todo en maneo, permaneo y remaneo la permanencia forma parte de uno de sus significados prevalentes. Ahora bien, como se ha visto en (1a)-(1c), esa permanencia no es siempre atélica; si lo es, ello puede estar marcado por elementos léxicos adicionales al contexto.

En definitiva, el estudio pormenorizado de los casos que han sido objeto de investigación en este trabajo ha permitido (i) dar cuenta de las situaciones específicas que ellos posibilitan, así como (ii) explicar y clarificar las causas y condiciones de esas situaciones.

Como conclusión adicional, es necesario señalar que el estudio de corpus ha servido de ayuda, cuando menos, para completar y matizar afirmaciones o posturas generales que en ocasiones se revelan necesitadas de una explicitación mayor.

\section{Bibliografía}

Cabrillana, C. (2010). Praedicativum and Subject Complement. In P. Anreiter, \& M. Kienpointner (Eds.), Latin Linguistics Today (pp. 233-245). Innsbruck: Innsbrucker Beiträge zur Sprachwissenschaft.

Cabrillana, C. (2015). The argument/satellite distinction and absolute verbal use in Latin stative verbs. Journal of Latin Linguistics, 14(2), 197-239.

Cabrillana, C. (2018). La exploración de concomitancias sintáctico-semánticas como vía de caracterización verbal en la lengua latina. Latomus, 77(3), 609-627.

Cabrillana, C. (2019a). Predicaciones secundarias: panorama de investigación y vías de análisis para la lengua latina. Comunicación presentada en el XLVIII Simposio de la Sociedad Española de Lingüistica, Madrid, 29/01-01/02/2019.

Cabrillana, C. (2019b). Constituent Order in Constructions with Praedicativum in Latin. Comunicación aceptada en el $20^{\text {th }}$ International Colloquium on Latin Linguistics, Las Palmas de Gran Canaria, 17-21/06/2019.

De Miguel, E., \& Fernández Lagunilla, M. (2002). Predicación secundaria y modificación adverbial. In A. Bernabé Pajares (Ed.), Actas del II Congreso de la Sociedad Española de Lingüistica (Madrid 11-15 diciembre 2000) (Vol. II; pp. 218-225). Madrid: Gredos. 
Demonte, V., \& Masullo, P. J. (1999). La predicación: los complementos predicativos. In I. Bosque, \& V. Demonte (Dirs.), Gramática descriptiva de la lengua española (Vol. II; pp. 2461-2523). Madrid: Espasa.

Di Tullio, A. (2002). La inestable frontera entre atributos y predicativos. Verba, 29, 9-29.

Fugier, H. (1983). Le syntagme nominale en latin classique. In W. Haase et al. (Eds.), Aufstieg und Niedergang der römischen Welt (Vol. II, 29, 1; pp. 212-269). Berlin: De Gruyter.

Givón, T. (1990). Syntax: A Functional-Typological Introduction (Vol. I). Amsterdam: Benjamins.

Gumiel, S. (2005). Los complementos predicativos. Madrid: Arco/Libro.

Gumiel, S. (2008). Estructura argumental y predicación secundaria. Alcalá de Henares: Universidad de Alcalá de Henares.

Halliday, M. A. K. (1967). Notes on transitivity and theme in English, Part I. Journal of Linguistics, 3, 37-81.

Himmelmann, N. P., \& Schultze-Berndt, E. (2005). Issues in the syntax and semantics of participantoriented adjuncts: an introduction. In N. P. Himmelmann, \& E. Schultze-Berndt (Eds.), Secondary Predication and Adverbial Modification (pp. 1-67). Oxford: Oxford University Press.

Hofmann, J. B., \& Szantyr, A. (1965). Lateinische Syntax und Stilistik mit dem allgemeinen Teil der lateinischen Grammatik. München: C. H. Beck.

Kühner, R., \& Stegmann, C. (1912-1914). Ausführliche Grammatik der lateinischen Sprache, II: Satzlehre (2 Vols.). Hannover: Hahnsche Buchhandlung.

Martínez Linares, Ma A. (2003). Sobre predicativos condicionales, predicados estativos y cuestiones conexas. Estudios de Lingüistica. Universidad de Alicante, 17, 427-450.

Pinkster, H. (1983). Praedicativum. In H. Pinkster (Ed.), Latin Linguistics and Linguistic Theory (pp. 199-217). Amsterdam: Benjamins.

Pinkster, H. (1995). Sintaxis y Semántica del Latín. Madrid: Ediciones Clásicas.

Pinkster, H. (2015). The Oxford Latin Syntax, I: The Simple Clause. Oxford: Oxford University Press.

Pinkster, H. (en prep.). Secondary predicates. Cap. 21 de The Oxford Latin Syntax (Vol. II; versión pre-final, 07/2018).

Rothstein, S. D. (1983). The Syntactic Forms of Predication (diss.). Cambridge, Mass.: Massachusetts Institute of Technology.

Schultze-Berndt, E., \& Himmelmann, N. P. (2002). Depictive Secondary Predicates in Cross-Linguistic Perspective [retrieved 07.07.2018 from https://pdfs.semanticscholar.org/7697/b20ce33f6f18a55a15ef6c582ce6f1dea9bc.pdf].

Silvagni, F. (2018). Sobre la distinción entre Individuo/Estadio y su relación con ser y estar. Revista Española de Lingüistica, 48, 15-56.

Tarriño, E. (2009). El Adjetivo. In J. M. Baños (Coord.), Sintaxis del latín clásico (pp. 251-272). Madrid: Liceus.

Vester, E. (1983). Instrument and Manner Expressions in Latin. Van Gorcum: Assen.

This work can be used in accordance with the Creative Commons BY-SA 4.0 International license terms and conditions (https://creativecommons.org/licenses/by-sa/4.0/legalcode). This does not apply to works or elements (such as image or photographs) that are used in the work under a contractual license or exception or limitation to relevant rights. 
Concepción Cabrillana PhD / concepcion.cabrillana@usc.es

Dpto. de Filología Clásica, Francesa e Italiana

University of Santiago de Compostela, Faculty of Philology

Avda. de Castelao, s/n., 15782-Santiago de Compostela (La Coruña), Spain 